\title{
Delayed presentation of head and neck cancer patients during COVID-19 pandemic
}

\author{
Fırat Tevetoğlu ${ }^{1} \mathbb{D} \cdot$ Sinem Kara ${ }^{1} \cdot$ Chinara Aliyeva $^{1} \cdot$ Rafet Yıldırım $^{1} \cdot$ H. Murat Yener ${ }^{1}$
}

Received: 29 December 2020 / Accepted: 26 February 2021 / Published online: 6 March 2021

(c) The Author(s), under exclusive licence to Springer-Verlag GmbH, DE part of Springer Nature 2021

\begin{abstract}
Purpose Impact of COVID-19 pandemic on healthcare is huge. We intended to demonstrate how COVID-19 pandemic affected primary head and neck oncology patient's referral and admission to a tertiary center by comparing the retrospective patient data in March-September 2020 and the same period in 2019.

Methods In this cross-sectional study, from March 15th, 2020 to September 15th, 2020, medical records of 61 patients (Group 1) diagnosed and scheduled for surgery for head and neck cancer in our tertiary care center were revised and compared with 64 head and neck cancer patients treated in the same institution in the same time period of the previous year (Group2). Surgical site, TNM stages, need for reconstruction with flap, time from first symptom occurrence to first admission to our institution, and time to surgery were noted.

Results In Group 1, out of 56 patients, 26 were diagnosed with T1-2 tumor, while 30 had T3-4 tumor. In Group 2, 43 of 60 patients had T1-2 tumor, while only 17 of them were diagnosed with T3-4 tumor. The rate of T3-4 tumors had significantly increased in 2020 when compared to $2019(p=0.049)$. In oral cavity cancer patients, $\mathrm{N}$ stage was significantly increased in Group 1 when compared to Group $2(p=0.024)$. Need for reconstruction with regional or free flaps were significantly increased in oral cavity cancer patients $(p=0,022)$. The mean time from the beginning of the first symptom to the admission was $19.01 \pm 4.6$ weeks (ranging between 11 and 32 weeks) in Group 1, while it was 16.6 \pm 5.9 weeks in Group 2 (ranging between 6 and18 weeks); with significant increase $(p=0,02)$. The time to surgery from first admission was $3.4 \pm 2.5$ and $2.9 \pm 1.2$ weeks in Group 1 and 2, respectively, with no statistically significant difference $(p=0.06)$.

Conclusion The COVID-19 pandemic has caused delay in the diagnosis and treatment of many diseases as such in head and neck cancers. Admission with advanced stage disease and the need for more complex reconstructive procedures were increased. During the pandemic, the management of other diseases that cause mortality and morbidity should not be neglected and priorities should be determined.
\end{abstract}

Keywords COVID-19 $\cdot$ Otolaryngology $\cdot$ Head and neck cancers $\cdot$ Delay

\section{Introduction}

Since the Coronavirus Disease 2019 (COVID-19) outbreak following first human infection on December 2019, many guidelines have been proposed on the management of COVID-19 patients. Apart from medical considerations, social and ethical burdens are enforcing the health care providers and pose dilemma on how to perform routine practice.

Firat Tevetoğlu

firattevetoglu@gmail.com

1 Cerrahpasa Medical Faculty, Otorhinolaryngology-Head and Neck Surgery Department, Istanbul University-

Cerrahpasa, Kocamustafapasa, Fatih, 34098 Istanbul, Turkey
Head and neck oncologic surgery should be scheduled timely due to several reasons. For good oncologic outcome, the treatment must be quickly planned and applied. It is well known that head and neck cancers increase in volume within 1-3 months [1]. Also due to potential of airway compromise and bleeding, these patients are to be treated without delay. On the other hand, cancer patients are more susceptible to infection as the malignancy itself and surgical or nonsurgical treatment entail systemic immune suppression [2].

Delay in diagnosis and treatment of head and neck cancer may result in upstage of the disease [3]. This may be either due to fear of the patients to apply to hospitals or overload of the hospitals, especially during pandemics. Guidelines recommend to perform oncologic surgeries 
within no more than 8 weeks during COVID-19 pandemics [4]. However, during this period, delay in treatment either due to patient or health system factors may affect the outcome of the patients seriously.

In this study, we intended to demonstrate how COVID19 pandemics affected primary head and neck oncology patient's referral and admission to a tertiary center by comparing the retrospective patient data in March-September 2020 and the same period in 2019.

\section{Materials and methods}

\section{Patient selection and data}

In this cross-sectional study, from March 15th, 2020 to September 15th, 2020, medical records of 61 patients diagnosed and scheduled for surgery due to head and neck cancer in our tertiary care center were revised and compared with 64 head and neck cancer patients treated in the same institution in the same time period of the previous year. The study has been approved by the institutional ethical review board.

Patients with paranasal sinus malignancies were excluded due to low number. Since the paper was structured mainly to evaluate the effects of COVID-19 pandemic on surgical treatment, oropharyngeal and hypopharyngeal carcinoma were also excluded for being treated with (chemo) radiotherapy primarily.

Group 1 (cases of 2020) was composed of 56 patients and Group 2 (cases of 2019) of 60 patients. During COVID-19 era, all of the patients in Group 1 were screened for COVID19 by history, nasal and oropharyngeal swap for PCR and thorax CT preoperatively.

All of the patients following proper preoperative head and neck examination were staged according to 8th Edition of AJCC (American Joint Committee on Cancer) TNM Classification. Also, time from first symptom occurrence to first admission to our institution and to surgery was recorded. The patients in both groups were surgically treated and need for flaps for reconstruction during surgery were noted. At the day of the operation, the patients were re-evaluated, and if no symptoms for COVID-19 was found, the surgery with appropriate precautions was commenced.

\section{Statistical analysis}

The statistical analysis was done with SPSS 21 program. The comparisons were done with Mann-Whitney $U$ test for numerical data and with Chi-square test for categorical data. $p<0.05$ was considered significant.

\section{Results}

The demographics, surgical site, TNM stages, need for reconstruction with flap, and time from first symptom occurrence to first admission to our institution and to surgery are outlined in Table 1 . Group 1 was composed of 56 patients ( 8 female and 48 male) with ages ranging between 43 and 77 years with a mean of $61.2 \pm 8.3$ years. 60 patients ( 11 female and 49 male) were included in Group 2; ages ranging between 28 and 72 with a mean of $59.6 \pm 7.7$ years. In Group 1, 36 patients were operated for laryngeal cancer and 20 patients for oral cavity cancer. In 2019, 35 patients were operated for laryngeal cancer and 25 patients for oral cavity cancer.

In Group 1, out of 56 patients 26 were diagnosed with T1-2 tumor, while 30 had T3-4 tumor. In Group 2, 43 of 64 patients had T1-2 tumor, while only 17 of them were diagnosed with T3-4 tumor, revealing that rates of T3-4 tumors had significantly increased in 2020 when compared to 2019 ( $p=0.049$ ). When data were further evaluated according to the subsites; out of 36 laryngeal cancer patients in Group 1, 17 patients had T1-2 tumor and 19 patients had T3-4 tumor; while 25 of 35 laryngeal cancer patients in Group 2 were diagnosed with T1-2 tumor and only 10 of them with T3-4 tumor. Again, this increase was found statistically significant $(p=0.048)$. For oral cavity cancer $\mathrm{T}$ stage, even the number of the patients with advanced stage disease was increased, this difference was not found statistically significant (Group 1: T1-2=9, T3-4=11; Group 2: T1-2=18, T3-4=7). In oral cavity cancer patients, $\mathrm{N}$ stage was significantly increased in Group 1 when compared to Group $2(p=0.024) .12$ oral cavity cancer patients out of 20 in Group 1 had N-positive disease and 8 had N0 disease, while 10 of 25 patients were diagnosed with $\mathrm{N}$-positive disease and 15 patients had N0 disease in Group 2. For N stage, no significant difference was found in laryngeal cancer patients or head and neck cancer patients in general.

Need for reconstruction with pedicled myocutaneous flap or composite free flap was significantly increased in oral cavity cancer patients $(p=0.022)$. Out of 20 patients operated for oral cavity cancer in 2020,8 of them necessitated reconstruction with flap, while only 5 of 25 patients operated in 2019 had flap reconstruction procedure.

The mean time from the beginning of the first symptom to the admission was $19.01 \pm 4.6$ weeks (ranging between 11 and 32 weeks) in Group 1, while it was 16.6 \pm 5.9 weeks in Group 2 (ranging between 6 and 18 weeks), with significant increase $(p=0.02)$. The time to surgery from first admission was $3.4 \pm 2.5$ and $2.9 \pm 1.2$ weeks in Group 1 and 2, respectively (both ranging between 1 and 6 weeks), with no significant difference $(p=0.06)$. 
Table 1 Demographic data, $\mathrm{T}$ and $\mathrm{N}$ stage, need for flap reconstruction, and mean time from the first symptom to admission and from admission to surgery

\begin{tabular}{|c|c|c|c|}
\hline & $\begin{array}{l}\text { Group } 1(2020) \\
(n=56, \mathrm{f} / \mathrm{m}: 8 / 48)\end{array}$ & $\begin{array}{l}\text { Group } 2(2019) \\
(n=60, \mathrm{f} / \mathrm{m}: 11 / 49)\end{array}$ & $p$ value* \\
\hline Oral cavity & 20 & 25 & \\
\hline Age & $64.2 \pm 10.01$ & $60.3 \pm 8.6$ & $0.14^{\mathrm{a}}$ \\
\hline T1-2 & 9 & 18 & $0.52^{\mathrm{b}}$ \\
\hline T3-4 & 11 & 7 & \\
\hline $\mathrm{N}+$ & 12 & 10 & $0.024^{b}$ \\
\hline Flap & 8 & 5 & $0.022^{b}$ \\
\hline Time from first symptom to admission (weeks) & $12-32 ; 19.8 \pm 5.1$ & $6-28 ; 16.44 \pm 6.6$ & $\mathbf{0 . 0 3}^{\mathrm{a}}$ \\
\hline Time from admission to surgery (weeks) & $1-6 ; 4.7 \pm 1.4$ & $1-6 ; 2.89 \pm 1.22$ & $0.26^{\mathrm{a}}$ \\
\hline Larynx & 36 & 35 & \\
\hline Age & $61.6 \pm 8.8$ & $58.9 \pm 6.7$ & $0.15^{\mathrm{a}}$ \\
\hline $\mathrm{T} 1-2$ & 17 & 25 & $\mathbf{0 . 0 4 8}^{\mathrm{b}}$ \\
\hline $\mathrm{T} 3-4$ & 19 & 10 & \\
\hline $\mathrm{N}+$ & 8 & 6 & $0.6^{\mathrm{b}}$ \\
\hline Flap & 1 & 1 & $0.9^{\mathrm{b}}$ \\
\hline Time from first symptom to admission (weeks) & $11-28 ; 18.5 \pm 4.3$ & $8-28 ; 17 \pm 5.3$ & $0.22^{\mathrm{a}}$ \\
\hline Time from admission to surgery (weeks) & $2-7 ; 3.4 \pm 1.2$ & $1-6 ; 2.9 \pm 1.1$ & $0.08^{\mathrm{a}}$ \\
\hline Total $^{2}$ & 56 & 60 & \\
\hline Age & $61.2 \pm 8.3$ & $59.6 \pm 7.7$ & $0.47^{\mathrm{a}}$ \\
\hline $\mathrm{T} 1-2$ & 26 & 43 & $0.049^{b}$ \\
\hline T3-4 & 30 & 17 & \\
\hline $\mathrm{N}+$ & 20 & 16 & $0.29^{\mathrm{b}}$ \\
\hline Flap & 9 & 6 & $0.19^{\mathrm{b}}$ \\
\hline Time from first symptom to admission (weeks) & $11-32 ; 19.01 \pm 4.6$ & $6-18 ; 16.6 \pm 5.9$ & $0.02^{\mathrm{a}}$ \\
\hline Time from admission to surgery (weeks) & $1-6 ; 3.4 \pm 2.5$ & $1-6 ; 2.9 \pm 1.2$ & $0.06^{\mathrm{a}}$ \\
\hline
\end{tabular}

*Statistically significant $\mathrm{p}$ values $(p<0.05)$ were shown as bold

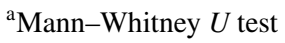

${ }^{\mathrm{b}}$ Chi-square test
Two of the laryngeal carcinoma patients had COVID-19 infection in preoperative period causing treatment delay. One of the oral cavity cancer patients was also diagnosed with COVID-19 infection in the post-operative period following discharge.

\section{Discussion}

The pandemic is causing increased demand on health care and special equipment, and the physicians are trying to balance themselves on managing critically ill patients and other patients seeking medical attention for diseases as malignancies. Since most of the resources are allocated to COVID-19, diagnosis and treatment of such diseases may delay which may be attributed to fear of the patients to apply to hospitals or overload of the hospitals. Delays in head and neck cancer care may also be due to derangement of tumor board meetings or need for quarantine of the patients, caregivers, or health care stuff [3].
Head and neck oncologic surgery should be scheduled timely due to increase in tumor volume within 1-3 months, potential of airway compromise and risk of severe bleeding [1] and delay in management may result in disease progression [3]. Data to date suggest that prolonged interval between surgery and post-operative radiation and delayed treatment initiation have a significant impact on overall survival [5, 5]. Although we were not able to assess survival in this report due to short follow-up period, we observed an increase in the advanced stage disease for head and neck cancer patients in our center, during COVID-19 pandemic. Overall rates of T3-4 tumors were significantly increased in 2020 when compared to 2019. Also, in oral cavity but not in larynx cancer, $\mathrm{N}$ stage was significantly increased in 2020 . These findings demonstrated that more head and neck cancer patients apply to hospitals with more locoregionally advanced disease. In 2020, admission time to hospital was also significantly increased demonstrating that patients were more reluctant to apply to hospitals no matter how severe the condition was. Our other observation was the increased need for reconstruction with pedicled myocutaneous or composite free flaps, 
especially in oral cavity cancer patients, due to larger excisions, which could be considered as a natural consequence of higher TNM stage.

In a recently published review, consensus was reached on not delaying the surgery more than 8 weeks for early stage (stage I-II) cancer, while maximum 4 weeks of delay was acceptable for advanced stage (stage III-IV) cancer [4]. However, in a study conducted by Murphy, it was shown that early stage cancers benefit more from the early surgery when compared to advanced stage, in means of survival [7]. Yet, we can conclude that surgery should not be delayed more than 8 weeks for head and neck cancer patients.

During natural disasters, it is well known that patients often ignore their symptoms [8]. One of the few studies about this problem was conducted by Ozaki who observed treatment delay in breast cancer patients following Fukushima incidence. Ozaki et al. [9] in our study, we observed that the mean time from the beginning of the first symptom to the time of surgery was significantly increased during pandemic. Although time to first admission to surgery was also increased during COVID-19 pandemic, the comparison result was insignificant. The longer duration of treatment initiation in this study was mostly due to delay in admission and partly due to increased time for preoperative preparations.

A review conducted by Han suggested a change in the traditional paradigm of evaluating the head and cancer patient as relying more on the imaging and minimally invasive procedures [8]. It was also stated that although surgery was the primary treatment modality for most head and neck cancers, nonsurgical alternatives should be taken into consideration to prevent treatment delays and the risk of nosocomial spread of SARS-CoV-2 during and after surgery [8]. We also believe that different measures such as increased public awareness about head and neck cancer symptoms or to establish COVID-19 free healthcare facilities must be considered to prevent delay in cancer treatment.

One of the drawbacks of our study was the lack of longterm follow-up of patients operated in 2020, so we were not able to compare survival or recurrence. Yet, in following years, real impact of COVID-19 pandemic will be appreciated by evaluating the survival and recurrence rates, which can also be a subject to another study. Another limitation is the subjectivity of the elongation of the time from first symptom to admission as it is a highly patient-dependent variable. This study lacks information about the primary (chemo) radiotherapy and adjuvant treatment, as our main objective was delay in diagnosis and treatment, upstaging of the disease, and increase of the need for complex reconstructive surgeries in patients treated surgically, which could also be considered as another drawback.

\section{Conclusion}

The COVID-19 pandemic caused delay in the diagnosis and treatment of many diseases including head and neck cancers. In our study, admissions with advanced stage disease and the need for more complex reconstructive procedures were found to be increased due to the delay in admission and partially due to the disruption caused by the allocation of most of the resources to the pandemic. This was seen in nearby effects such as larger resections and more sophisticated reconstructions but overall impacts such as survival and long-term care are yet not appreciated. During the pandemic, we believe that management of other diseases that cause mortality and morbidity should not be neglected and priorities should be determined. If necessary, establishment of uncontaminated centers that carry out the diagnosis and treatment of the diseases other than COVID-19 should be considered.

Funding This research received no specific grant from any funding agency, commercial, or not-for-profit sectors.

Availability of data and materials The data of the study can be shared on demand.

Code availability Not applicable.

\section{Declarations}

Conflict of interest The authors declare no conflict of interest.

Ethical considerations Informed consent was obtained from all of the patients. Study design has been submitted for approval of ethics committee. There is no disclosure of personal information and images of patients in this paper.

Consent for publication All the patients and all the authors consented the publication of the data.

Consent to participate All participants were informed and informed consents were retrieved.

\section{References}

1. Jensen AR, Nellemann HM, Overgaard J (2007) Tumor progression in waiting time for radiotherapy in head and neck cancer. Radiother Oncol 84(1):5-10. https://doi.org/10.1016/j.radon c. 2007.04 .001

2. M. C. Topf et al., "A Framework for Prioritizing Head and Neck Surgery during the COVID-19 Pandemic.,"Head Neck, p. hed.26184, Apr. 2020, https://doi.org/10.1002/hed.26184.

3. Werner MT, Carey RM, Albergotti WG, Lukens JN, Brody RM (2020) Impact of the COVID-19 Pandemic on the Management of Head and Neck Malignancies. Otolaryngol Head Neck Surg 
(United States) 162(6):816-817. https://doi.org/10.1177/01945 99820921413

4. H. Mehanna et al., Recommendations for head and neck surgical oncology practice in a setting of acute severe resource constraint during the COVID-19 pandemic: an international consensus. Lancet Oncol, vol. 21, no. 7. Lancet Publishing Group, pp. e350 e359, 01-Jul-2020, https://doi.org/10.1016/S1470-2045(20)30334 $-\mathrm{X}$.

5. H. W. Schutte et al., Impact of Time to Diagnosis and Treatment in Head and Neck Cancer: A Systematic Review. Otolaryngology Head Neck Surgery (United States), vol. 162, no. 4. SAGE Publications Inc., pp. 446-457, Apr-2020, https://doi. org/10.1177/0194599820906387.

6. E. M. Graboyes et al., Association of Treatment Delays with Survival for Patients with Head and Neck Cancer: A Systematic Review. JAMA Otolaryngology Head Neck Surgery, vol. 145, no. 2. American Medical Association, pp. 166-177, 01-Feb-2019, https://doi.org/10.1001/jamaoto.2018.2716.

7. Murphy CT et al (2016) Survival impact of increasing time to treatment initiation for patients with head and neck cancer in the United States. J Clin Oncol 34(2):169-178. https://doi. org/10.1200/JCO.2015.61.5906

8. A. Y. Han, J. E. Miller, J. L. Long, and M. A. St John, Time for a Paradigm Shift in Head and Neck Cancer Management During the COVID-19 Pandemic. Otolaryngol Head Neck Surg. (United States), vol. 163, no. 3, pp. 447-454, Sep. 2020, https://doi. org/10.1177/0194599820931789.

9. Ozaki A et al (2017) Breast cancer patient delay in Fukushima, Japan following the 2011 triple disaster: A long-term retrospective study. BMC Cancer 17(1):423. https://doi.org/10.1186/s1288 5-017-3412-4

Publisher's Note Springer Nature remains neutral with regard to jurisdictional claims in published maps and institutional affiliations. 